\title{
Evaluation of Fabry-Perot polymer film sensors made using hard dielectric mirror deposition
}

Buchmann, Jens, Zhang, Edward, Scharfenorth, Chris, Spannekrebs, Bastian, Villringer, Claus, et al.

Jens Buchmann, Edward Zhang, Chris Scharfenorth, Bastian Spannekrebs, Claus Villringer, Jan Laufer, "Evaluation of Fabry-Perot polymer film sensors made using hard dielectric mirror deposition," Proc. SPIE 9708, Photons Plus Ultrasound: Imaging and Sensing 2016, 970856 (15 March 2016); doi: $10.1117 / 12.2234698$

SPIE. Event: SPIE BiOS, 2016, San Francisco, California, United States 


\title{
Evaluation of Fabry-Perot polymer film sensors made using hard dielectric mirror deposition
}

\author{
Jens Buchmann ${ }^{\mathrm{a}}$, Edward Zhang ${ }^{\mathrm{b}}$, Chris Scharfenorth $^{\mathrm{a}}$, Bastian Spannekrebs ${ }^{\mathrm{a}}$, \\ Claus Villringer ${ }^{\mathrm{d}}$, Jan Laufer ${ }^{\mathrm{a}, \mathrm{c}}$ \\ anstitute of Optics and Atomic Physics, Technical University Berlin, Straße des 17. Juni 135, \\ 10623 Berlin, Germany \\ ${ }^{b}$ Department of Medical Physics and Bioengineering, University College London, Gower Street, \\ London WC1E 6BT, UK \\ 'Institute of Radiology, Charité University Hospital, Charitéplatz 1, 10117 Berlin, Germany \\ ${ }^{\mathrm{d} U n i v e r s i t y ~ o f ~ A p p l i e d ~ S c i e n c e s ~ W i l d a u, ~ H o c h s c h u l r i n g ~ 1, ~} 15745$ Wildau, Germany
}

\begin{abstract}
Fabry-Perot (FP) polymer film sensors offer high acoustic sensitivity, small element sizes, broadband frequency response and optical transmission to enable high resolution, backward mode photoacoustic (PA) imaging. Typical approaches to sensor fabrication involve the deposition of stacks of alternating dielectric materials to form interferometer mirrors, which are separated by a polymer spacer. If hygroscopic soft dielectric materials are used, a protective polymer layer is typically required. In this study, methods for the deposition of water-resistant, hard dielectric materials onto polymers were explored to improve the robustness and performance of the sensors. This involved the optimisation of the fabrication process, the optical and acoustic characterisation of the sensors, and a comparison of the frequency response with the output of an acoustic forward model. The mirrors, which were separated by a $20 \mu \mathrm{m}$ Parylene spacer, consisted of eight double layers of $\mathrm{Ta}_{2} \mathrm{O}_{5}$ and $\mathrm{SiO}_{2}$ deposited onto polymer substrates using temperature-optimised electron vapour deposition. The free spectral range of the interferometer was $32 \mathrm{~nm}$, its finesse $F_{R}=91$, and its visibility $V=0.72$. The noise-equivalent pressure was $0.3 \mathrm{kPa}(20 \mathrm{MHz}$ bandwidth). The measured frequency response was found to be more resonant at $25 \mathrm{MHz}$ compared to sensors with soft dielectric mirrors, which was also in good agreement with the output of a forward model of the sensor. The sensors were used in a PA scanner to acquire 3-D images in tissue phantoms.
\end{abstract}

Keywords: Photoacoustic tomography, Fabry Perot, interferometer

\section{INTRODUCTION}

Photoacoustic tomography (PAT) allows non-invasive imaging of vasculature to cm-depths. It typically involves the illumination of biological tissue with nanosecond laser pulses to generate broadband ultrasound waves up to tens of MHz. The ideal ultrasound detector for high resolution imaging should provide a) high acoustic sensitivity, b) small active area size, c) broadband frequency response, and d) transparency to the excitation wavelength to allow backward mode imaging. Conventional piezoelectric receivers do not meet all of these requirements as they usually offer limited bandwidth due to the resonant frequency response while the sensitivity reduces with diminishing active element size. Optical ultrasound detectors, such as Fabry-Perot (FP) polymer film sensors and microring resonators ${ }^{1,2}$ have been shown to offer attractive alternatives. FP sensors in particular offer broadband frequency response, high sensitivity at small active area sizes as well as transparency to visible and near-infrared excitation pulses to enable photoacoustic imaging in backward mode ${ }^{3}$. FP sensors can be produced by deposition of alternating stacks of dielectric materials with different refractive index ${ }^{4}$ or by glancing angle deposition (GLAD) ${ }^{5}$. GLAD has been used to fabricate FP interferometers on glass substrates, thus limiting their acoustic bandwidth. By contrast, FP polymer film sensors have been made by depositing alternating high-/low-refractive soft dielectric materials, i.e. $\mathrm{ZnS}$ and $\mathrm{Na}_{3} \mathrm{AlF}_{6}{ }^{4}$, onto polymer

Photons Plus Ultrasound: Imaging and Sensing 2016, edited by Alexander A. Oraevsky, Lihong V. Wang Proc. of SPIE Vol. 9708, 970856 · @ 2016 SPIE · CCC code: 1605-7422/16/\$18 · doi: 10.1117/12.2234698 
substrates and spacers, which resulted in a broadband frequency response of up to $100 \mathrm{MHz}$ depending on spacer thickness. However, some soft dielectric materials are hygroscopic, which necessitates an additional protective polymer layer. In this study, the methods for the deposition of mirrors consisting of so-called hard dielectric materials onto polymer substrates are developed. The acoustic sensitivity and frequency response of the sensors is measured and their PA imaging capability demonstrated. The measured frequency response is compared to the output of a numerical model of the acoustic propagation through the sensor.

\section{METHODS}

\subsection{Sensor fabrication}

The dielectric mirrors of the FP sensor was designed such that they exhibited high transmission in the so-called NIR window of tissue (600-1000 nm), which allows backward mode biomedical PA imaging, and high reflectivity around $1550 \mathrm{~nm}$, which corresponds to the wavelength range of the tuneable interrogation laser. Sensor fabrication involved the deposition of stacked layers of dielectric materials to form the mirrors and a polymer spacer to form the interferometer cavity. The substrate consists of cyclic olefin polymer (ZEONEX 480R) with a diameter of $8 \mathrm{~cm}$ and a thickness of 1 $\mathrm{cm}$. Using electron vapour deposition, alternating $\lambda / 4$ thick layers of $\mathrm{SiO}_{2}$ and $\mathrm{Ta}_{2} \mathrm{O}_{5}$ were deposited onto the substrate to form the first mirror of the interferometer. The dielectric mirrors consist of a total of eight double layers. This was followed by chemical vapour deposition of a Parylene $C$ film of $20 \mu \mathrm{m}$ thickness and the deposition of the second dielectric mirror. Since the resulting interferometer mirrors exhibited significant optical attenuation in the Vis/NIR wavelength range, the finished sensors were tempered to improve their transparency.

\subsection{Experimental setup}

The experimental setup for 3-D PA imaging of phantoms and in vivo is shown in Figure 1 and is based on that described by Zhang et $\mathrm{al}^{4}$. The excitation laser is an Innolas Spitlight 1000 which consists of a Nd:YAG pump laser and a type II optical parametric oscillator (OPO) with continuous wavelength tuning capability from $420-2300 \mathrm{~nm}$ and repetition rate of $30 \mathrm{~Hz}$. The output of the OPO is coupled into a $1.5 \mathrm{~mm}$ multimode fibre to homogenise the beam. At the end of the fibre the beam is collimated and directed onto a dichroic mirror which directs the beam through the FP sensor and onto the sample. The sensor is read out by raster scanning the focused beam provided by a single model fibre-coupled tuneable diode laser (Yenista T100S).

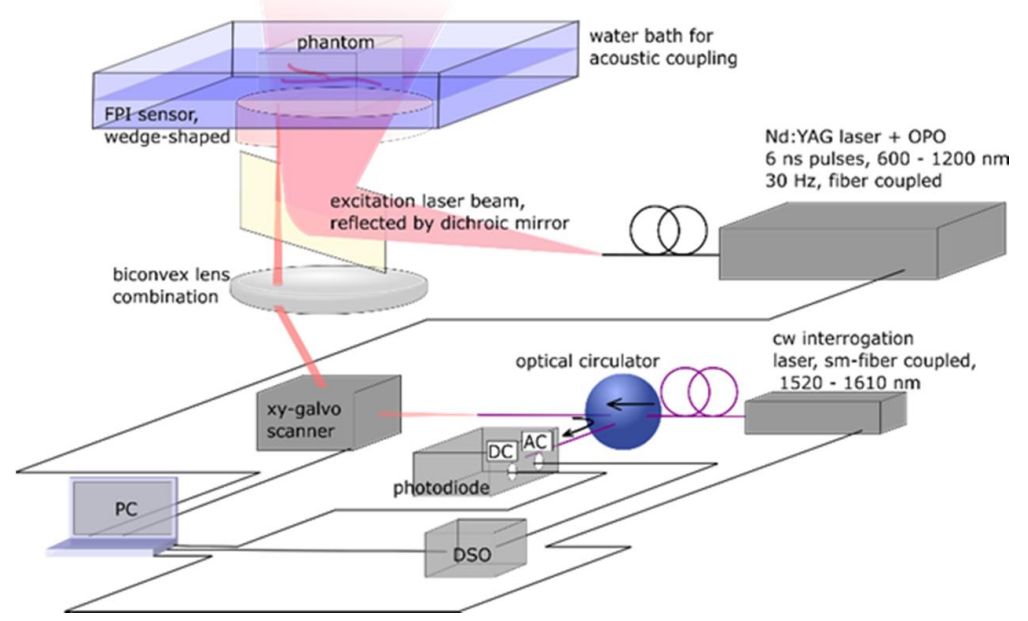

Figure 1. Experimental setup for PA tomography in backward-mode using the all-optical scanner based on a Fabry-Perot polymer film sensor.

Acoustic waves incident on the sensor modulate the optical thickness of the interferometer and induce a phase shift between the reflections from the interferometer mirrors, and hence the interferometer transfer function (ITF). By tuning 
the wavelength of the interrogation laser to the maximum slope of the ITF, this phase shift results in a change in the reflected optical power. This change is linear for acoustic transients of low intensity and the phase sensitivity is directly dependent upon the steepness of the ITF slope. The interrogation light reflected by the sensor is coupled back into the single-mode fibre and directed to a photodiode using an optical circulator. The output of the photodiode is connected to a digitizer card (National Instruments), which is used to acquire the time-resolved PA signals. By 2-D raster scanning the interrogation beam across the sensor, and by generating and acquiring PA signals at each scan position, the spatial and temporal distribution of PA waves can be mapped, which allows the subsequent reconstruction of the initial pressure distribution using image reconstruction algorithms ${ }^{6}$.

\subsection{Modelling the frequency response of the sensor}

An analytical model for the frequency response of Fabry-Perot polymer film sensors consisting of aluminium mirrors and a PET spacing layer was first developed by Beard et al. in $1999^{3}$. This model was subsequently validated for FabryPerot sensors with dielectric mirrors consisting of alternating stacks of $\mathrm{ZnS}$ and $\mathrm{Na}_{3} \mathrm{AlF}_{6}$ and a Parylene $\mathrm{C}$ polymer spacer $^{4}$.

A limitation of this model is that the thickness of the interferometer mirrors is either considered much smaller than the acoustic wavelength (e.g. in the case of metal mirrors) or that the acoustic impedance of the mirrors is sufficiently similar to polymers (e.g. in the case of soft dielectric mirrors) to allow their effect on the frequency response to be neglected. However, given the substantial acoustic impedance mismatches produced by hard dielectric materials compared to the polymer materials of the substrate and the spacer (see Table 1), this assumption may not be valid.

Table 1. Acoustic impedances and layer thicknesses of the materials used in the fabrication of Fabry-Perot sensors?

\begin{tabular}{ccccc}
\hline Material & Density $\left(\mathbf{k g} / \mathbf{m}^{\mathbf{3}}\right)$ & $\begin{array}{c}\text { Speed of sound } \\
(\mathbf{k m} / \mathbf{s})\end{array}$ & $\begin{array}{c}\text { Acoustic impedance } \\
\left(\mathbf{k g} / \mathbf{m}^{2} / \mathbf{s} * \mathbf{1 0}^{\mathbf{6}}\right)\end{array}$ & $\begin{array}{c}\text { Layer } \\
\text { thickness }\end{array}$ \\
\hline COP 480R & 1010 & 2.7 & 2.73 & $1 \mathrm{~cm}$ \\
\hline Parylene & 1289 & 2.2 & 2.84 & $20 \mu \mathrm{m}$ \\
\hline $\mathbf{S i O}_{2}$ & 2200 & 6 & 13.2 & $246 \mathrm{~nm}$ \\
\hline $\mathbf{T a}_{2} \mathbf{O}_{5}$ & 8200 & 5 & 41 & $175 \mathrm{~nm}$ \\
\hline
\end{tabular}

In this study, a 1-D numerical model of the sensor was implemented in k-Wave ${ }^{6}$ to calculate the frequency response of sensors made using hard dielectric mirror deposition. The model input parameters are the spatial distribution of acoustic properties of the sensor materials (Table 1) and the acoustic coupling medium of the sensor (water). An initial pressure distribution was modelled as a Gaussian distribution $(\mathrm{FWHM}=5 \mu \mathrm{m})$ and located adjacent to the sensor to ensure a broadband acoustic frequency spectrum similar to that observed in PA waves.

The acoustic propagation of the initial pressure distribution results in the propagation of two acoustic waves in $+x$ and $-x$ directions, each with half of the initial pressure amplitude. When the wave propagating into $-x$ direction traverses the sensor, multiple reflections occur due to the impedance mismatches between the dielectric mirrors, the polymers, and the water. This affects the acoustic field, and therefore also the time-resolved PA signal that is recorded by the sensor.

It was assumed that the change in the optical thickness of the hard dielectric mirror materials is negligible compared to that induced in the Parylene spacer. The acoustic signal, $R_{1}(t)$, detected by the sensor was obtained by integrating the time-varying pressure field over the thickness of the Parylene spacer for each time step. The frequency response of the sensor can then be obtained by calculating the Fast Fourier Transform (FFT) of $R_{1}(t)$ and by dividing it by the FFT of a signal $R_{2}(t)$, which was obtained for an ideal, non-band-limited single point detector in an acoustically homogenous medium. 


\subsection{Experimental measurement of the frequency response}

In order to obtain the frequency response of the hard dielectric FP sensors from measured data, a reference sensor of known frequency response was used. A FP sensor with soft dielectric mirrors ${ }^{4}$ with a $20 \mu$ m Parylene spacing layer was used for this purpose as its frequency response closely resembles that of the analytical model ${ }^{3}$.

A planar, broadband PA wave was generated in an India ink solution $\left(\mu_{\mathrm{a}}=540 \mathrm{~cm}^{-1}\right)$ using the output of the OPO lasers as shown in Figure 2. The ink was placed in a polymer (PET) petri dish and deionized water was used for acoustic coupling to the FP sensor. The excitation laser beam $(1064 \mathrm{~nm}, 3 \mathrm{~mJ})$ was collimated by two plano-convex lenses to produce a beam diameter of $2 \mathrm{~cm}$ at the sample. The measurements were made in forward mode.

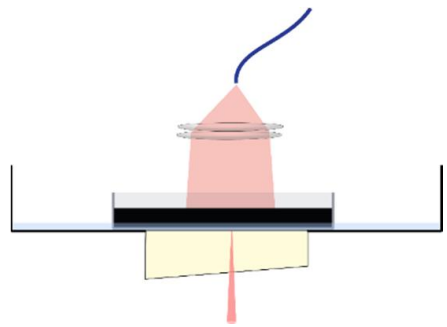

Figure 2. Experimental setup for the measurement of the frequency response. A solution of India ink was illuminated by a large diameter collimated beam.

PA signals were recorded at a single point with a Tektronix DPO 5034 oscilloscope with a time resolution of 200 ps. Signals were averaged over 500 pulses. Prior to every measurement, the India ink solution was stirred to prevent particle sedimentation, which was found to affect the homogeneity of the absorption coefficient especially near the surface of the liquid. PA signals were recorded using the reference (soft dielectric) FP sensor and the hard dielectric FP sensor. The ratio of the Fourier transformations of the PA signals, and taking into account the known frequency response of the reference sensor, yielded the frequency response of the hard dielectric FP sensor.

\subsection{Determination of noise-equivalent pressure}

The noise-equivalent pressure (NEP) of a sensor is the pressure threshold where the signal amplitude is the same as that of the peak-to-peak noise. To determine the noise-equivalent pressure of the sensor, a PZT ultrasound transducer with a $23 \mathrm{~mm}$ dia. active area and $4 \mathrm{MHz}$ centre frequency (Precision Acoustics Ltd) was calibrated against the output of a 0.2 $\mathrm{mm}$ needle hydrophone (Precision Acoustics Ltd). The near field pressure of the transducer was calibrated with a $15 \%$ confidence interval. For the measurement of the noise-equivalent pressure, the transducer was placed a few millimetres above the sensor and acoustically coupled using deionized water as shown in Figure 3. The transducer was connected to a frequency generator (Agilent 33522A), which generated sine wave bursts with 5 periods at a frequency of $4 \mathrm{MHz}$, matching the centre frequency of the ultrasound transducer. Between every burst sufficient time was given for the acoustic waves to settle, avoiding the creation of standing waves and reflections. The FP sensor was interrogated at a single point at the centre of the pressure field emitted by the transducer. The NEP was measured over a bandwidth of 20 $\mathrm{MHz}$, which allows a comparison with literature data from sensors with soft dielectric mirrors ${ }^{4}$.

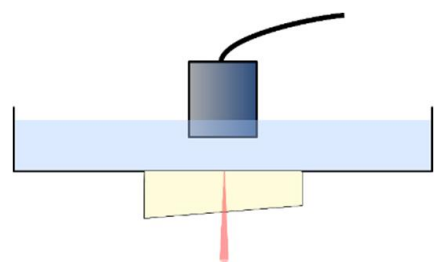

Figure 3. A calibrated ultrasound transducer with a centre frequency of $4 \mathrm{MHz}$ is used to produce an ultrasound tone burst in a water bath. The ultrasound waves are then measured using the FP sensor. 


\subsection{Tomographic measurements of tissue mimicking phantom}

To demonstrate the application of hard dielectric FP sensors to high resolution 3-D tomographic imaging, a tissuemimicking phantom was constructed. The phantom consisted of multiple crossed pairs of black nylon strings with a diameter of $70 \mu \mathrm{m}$ suspended in diluted milk (1,5\% milk, 30\% volume percent dilution with water) at different depths ranging from 4 to $15 \mathrm{~mm}$. The scattering coefficient of the milk dilution was not measured independently, but can be estimated to be in the range of $\mu_{\mathrm{s}}{ }^{\prime}=5-10 \mathrm{~cm}^{-1}{ }^{8}$, resembling a typical scattering coefficient found in biological tissue. A scan area of $15 \times 15 \mathrm{~mm}^{2}$ of the FP sensor was interrogated with step size of $150 \mu \mathrm{m}$. The excitation wavelength was 617 $\mathrm{nm}$ with a pulse energy of $8 \mathrm{~mJ}$ reaching the phantom, corresponding to a fluence of less than $4 \mathrm{~mJ} / \mathrm{cm}^{2}$. Reconstruction is performed using a Fourier-based reconstruction algorithm. Optical attenuation compensation $\left(\mu_{\mathrm{a}}=150 / \mathrm{cm}\right)$ and logarithmic compression of the reconstructed signal was used to improve the visibility of the deeper lying nylon strings.

\section{RESULTS}

\subsection{Interferometer characterisation and acoustic sensitivity}

A typical interferometer transfer function (ITF) of a hard dielectric FP sensor is shown in the left panel of Figure 4. The free spectral range of the interferometer is $32 \mathrm{~nm}$. A single fringe is shown in the centre panel of Figure 4 . A linear combination of Gauss and Lorentz functions (so-called pseudo-Voigt function) provided the best fit to the data. With a calculated mean fringe FWHM of $0.35 \mathrm{~nm}$ (over a 10x10 $\mathrm{mm}^{2}$ area), the finesse of the sensor it close to $100(\mathrm{~F}=91)$. The mean visibility is 0.71 . The mean sensitivity (maximum slope of the ITF, derived from the fitted pseudo-Voigt functions) over the same scan area is $0.71 \mathrm{~V} / \mathrm{nm}$. The histogram of the sensor sensitivity is shown in the right panel of Figure 4, which illustrates the homogeneous sensitivity provided by the sensor over a large scan area.
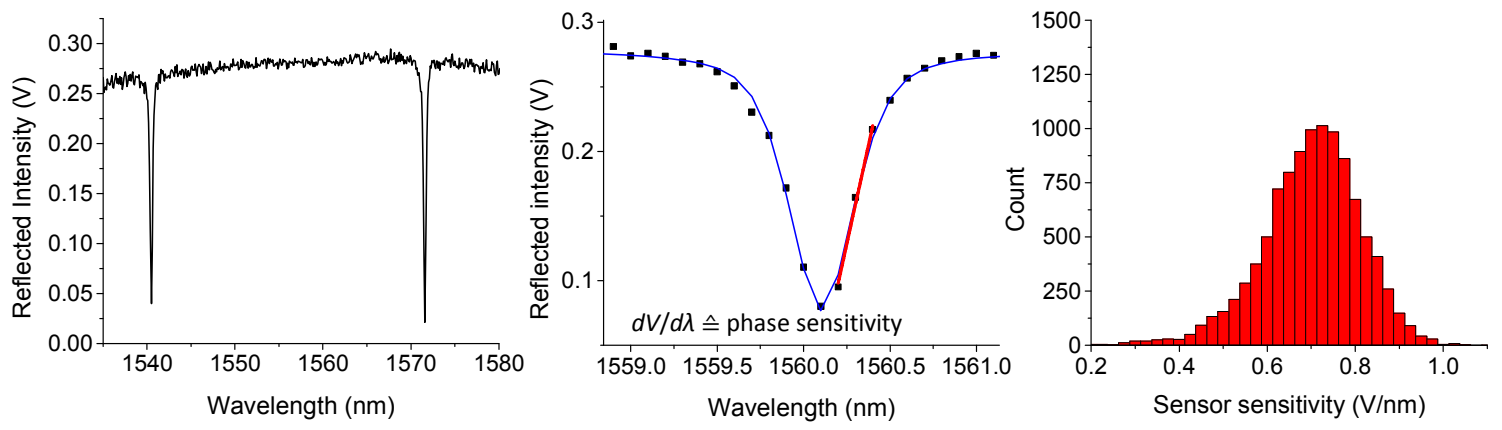

Figure 4. Characterization of the FP Sensor: Left: ITF of FP sensor. Middle: Single fringe and fitted a pseudo-Voigt function. Right: Distribution of sensitivity of single points over 2D scan area $\left(10 \times 10 \mathrm{~mm}^{2}, 0.1 \mathrm{~mm}\right.$ spacing, 10000 points in total).

The noise-equivalent-pressure was measured using the method described in section 2.4 and was found to be $0.33 \pm 0.07$ $\mathrm{kPa}$, which is comparable to previous sensor designs based on soft dielectric mirrors ${ }^{4}$.

\subsection{Frequency response}

The frequency response was modelled as described in section 2.2. A 1-D grid with $2^{15}=32768$ points was initialized, with a spacing of $5 \mathrm{~nm}$ between the grid points. The initial pressure distribution in water was modelled as a Gaussian function with a FWHM of $5 \mu \mathrm{m}$. The calculation was performed with time steps of $100 \mathrm{fs}$, which were needed to ensure stability and small phase errors within the very heterogeneous medium of the sensor. The model was run for a total of $10^{6}$ time steps $\left(t_{\text {end }}=1 \mu \mathrm{s}\right)$. In Figure 5, the modelled pressure distributions across the sensor are shown for different time points. It is noticeable that a significant fraction of the incoming pressure wave is reflected at the first mirror due to the high acoustic impedance mismatch, leading to a reduction in acoustic sensitivity. 

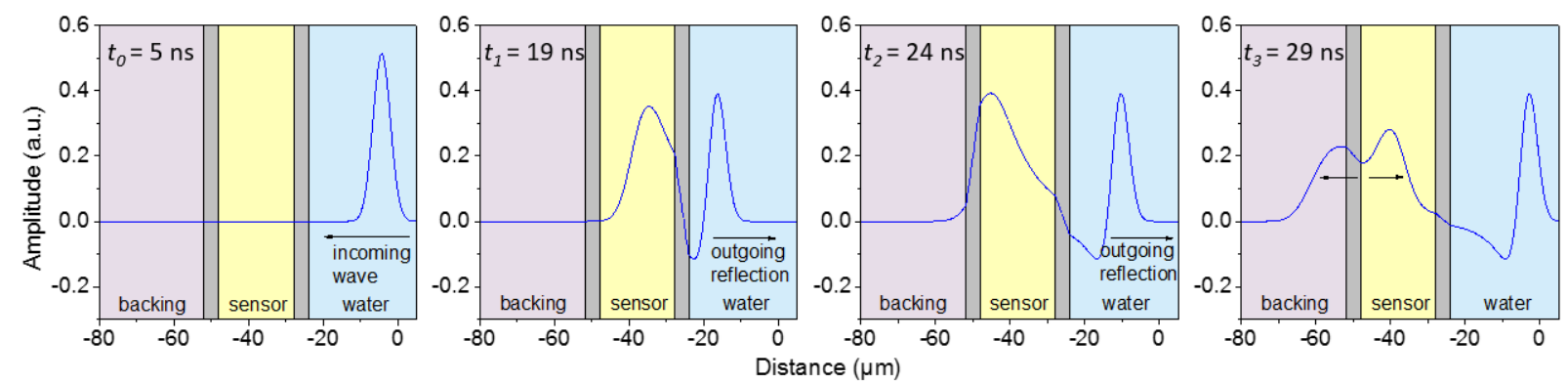

Figure 5. Pressure distribution across a FP sensor with hard dielectric mirrors at different points in time for an incident ultrasound wave showing reflections due to acoustic impedance mismatches.

The pressure distribution across the Parylene spacer was integrated for every time step. To reduce memory consumption, the pressure was recorded for 100 evenly spaced sample points across the Parylene spacer. The integration of the signal amplitude over all sample points leads to a time-dependent sensor output signal, $R_{1}(t)$. The reference signal, $R_{2}(t)$, was calculated using the one-sided solution of the wave equation for a Gaussian function as $R_{2}(t)=\mathrm{A} / 2\left[\exp \left(-\left(x-x_{0}+c t / \sigma\right)^{2}\right)\right]$ where $c$ is the speed of sound in water, $x_{0}$ is the location of the initial pressure distribution, $\sigma$ is the width of the Gaussian pulse, and $\mathrm{A}$ is a scaling parameter. The ratio of the Fourier transforms of the two signals, $\operatorname{FFT}\left(R_{1}(t)\right) / \operatorname{FFT}\left(R_{2}(t)\right)$, yields the modelled frequency response of the sensor. A simplified model was used to calculate the signal for a sensor with homogeneous acoustic properties, i.e. without dielectric mirrors, assuming the acoustic impedance of water for the first mirror and the acoustic impedance of the backing material for the second mirror. The corresponding frequency response was calculated as described above. The results obtained using the models are shown in the left panel of Figure 6 . Values for sensitivity are given in $\mathrm{dB}$ with the conversion $\mathrm{S}_{\mathrm{db}}(\mathrm{f})=10 \log _{10} \mathrm{~S}(\mathrm{f})$. The frequency response curves were normalized to the sensitivity at low frequencies. The right panel of Figure 6 shows the ratio of the two models. A resonant peak at around $25 \mathrm{MHz}(+5 \mathrm{~dB})$ is visible, which is due to the dielectric layers and the corresponding acoustic impedance mismatch. The results for the simplified model without accounting for the acoustic impedance mismatches of the mirrors is in perfect agreement with the analytical solution of the frequency response ${ }^{3}$ (data not shown).
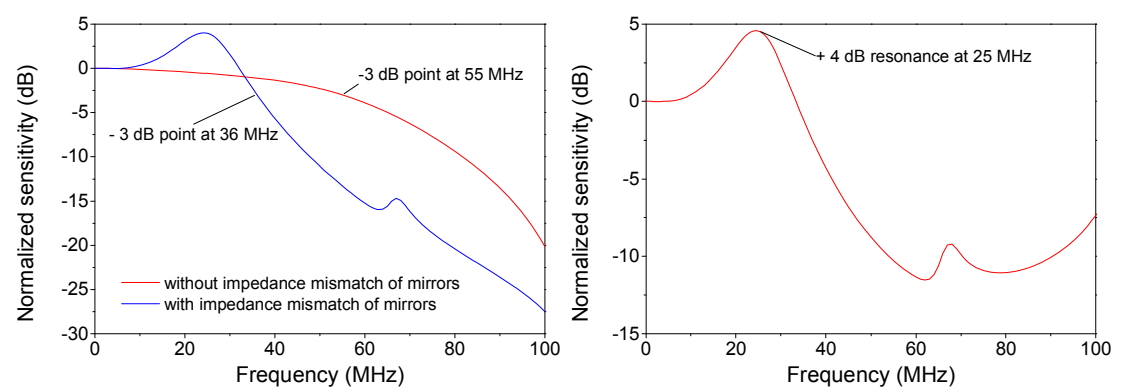

Figure 6. Modelled frequency response of FPI sensor. Left panel: with and without taking the hard dielectric mirror layers into account (normalized), Right panel: Ratio of both models showing a resonant peak at $25 \mathrm{MHz}$.

The modelled frequency response was also validated experimentally. PA waves generated in India ink were measured using the hard dielectric FP sensor and the soft dielectric FP sensor and are shown in Figure 7 together with their respective FFTs. The absorption coefficient of the India ink solution was determined by fitting an exponential function to the compressive part of the PA signal and was found to be $\mu_{\mathrm{a}}=540 / \mathrm{cm}$. In the signal recorded the hard dielectric FP sensor, the effect of acoustic reflections within the sensor are visible in the tensile part of the PA wave around $7.7 \mu \mathrm{s}$. By contrast, this oscillation is absent in the signal detected using the reference sensor based on soft dielectric mirrors. The FFT of the PA signal measured using the hard dielectric FP sensor exhibits a broader frequency response with higher values for frequencies in the range of $25 \mathrm{MHz}$ compared to that of the reference sensor. 
a)

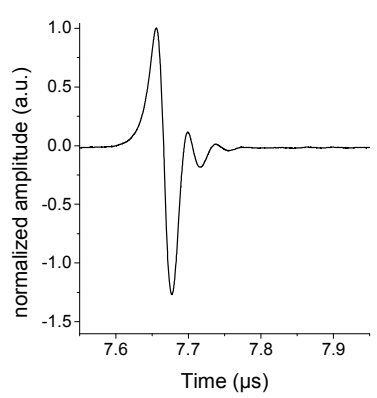

b)

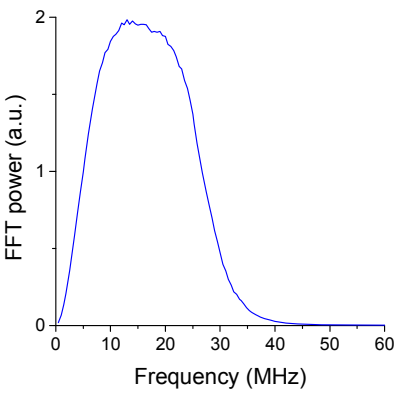

c)

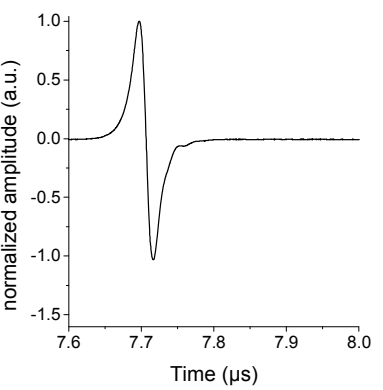

d)

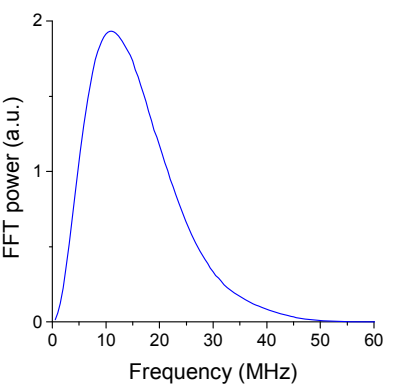

Figure 7. Frequency response of broadband PA signals generated in India ink. (a) PA signal measured using sensor with hard dielectric materials and (b) corresponding FFT, (c) PA signal measured using sensor with soft dielectric materials and (d) the corresponding FFT.

In Figure 8, the measured and modelled frequency responses of the hard dielectric FP sensor are shown. This was determined by first calculating the true frequency spectrum of the PA signals generated in India ink, i.e. by calculating the ratio of the Fourier spectrum of the measured PA signal and the known frequency response of the reference sensor. The Fourier spectrum of the PA signal acquired using the hard dielectric FP sensor was then divided by the true frequency spectrum of the PA signal to yield the frequency response of the sensor. The measured and modelled frequency responses were normalized with respect to sensitivity at low frequencies and are in good agreement. Both exhibit a resonant peak at $25 \mathrm{MHz}$ of comparable magnitude and agree well up to $40 \mathrm{MHz}$. At higher frequencies, the effects of measurement noise become more noticeable.

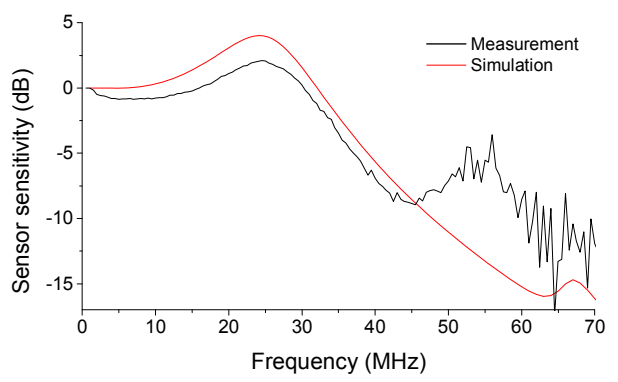

Figure 8. Comparison of the frequency response determined by experiment and model.

\subsection{D phantom imaging}

A photograph of the phantom used for imaging experiments is shown in the left panel of Figure 9. The maximum intensity projection (MIP) of the reconstructed 3-D image is shown in the right panel. The colour code represents image depth. All four nylon string pairs are visible in the reconstructed image, the deepest string is located at a depth of $15 \mathrm{~mm}$. The size of the nylon strings ( $70 \mu \mathrm{m}$ dia.) is too large for meaningful measurements of the maximum spatial resolution, but by taking axial and lateral profiles through the reconstructed image one can nevertheless obtain approximate values. 

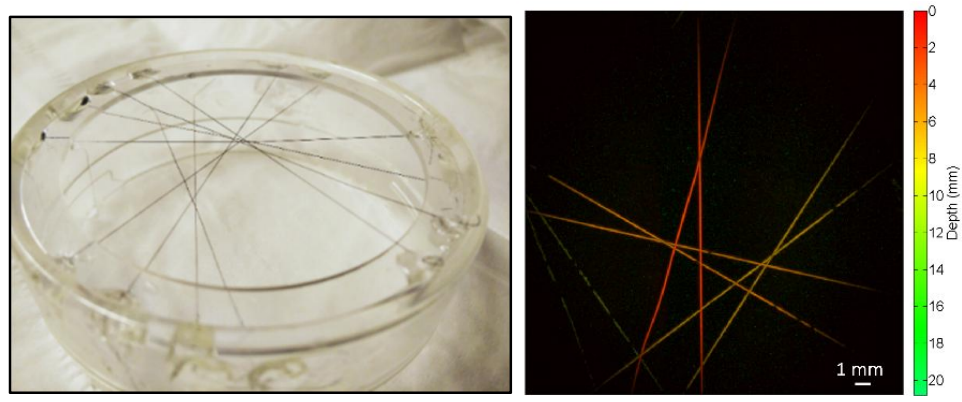

Figure 9. Left: Photograph of the tissue phantom, which consisted of crossed nylon strings at different depths, attached on a PET frame, and submerged in a scattering liquid. Right: Reconstructed 3-D image in maximum intensity projection (MIP), colours correspond to depth.

The two left panels of Figure 10 show single $z$-y-slices through the 3-D image. A pair of nylon strings at a depth of $4 \mathrm{~mm}$ are shown at two locations: 1) spatially separated and 2) in close proximity. The dashed lines indicate the location at which image intensity profiles were obtained, shown on the remaining panels of Figure 10. The lateral profile (1) exhibits a FWHM of $121 \mu \mathrm{m}$ (fitted with Gaussian), considerably wider than the thickness of the strings $(70 \pm 10 \mu \mathrm{m})$. This suggests a lateral resolution that is comparable to active element size defined by the diameter of the interrogation beam (approx. $40 \mu \mathrm{m}$ ). The axial resolution is shown in profiles (2) and (3). In profile (2) a Gaussian with FWHM of 59 $\mu \mathrm{m}$ was fitted to the photoacoustic signal, which agrees well to the diameter of the strings. In profile (3), the strings are in close proximity but can be distinguished clearly with FWHM values of $56 \mu \mathrm{m}$ and $71 \mu \mathrm{m}$. In order to quantify both axial and lateral resolutions of the sensor, a phantom with smaller features will be constructed for future measurements.

a)

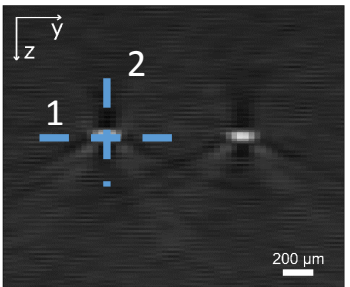

b)

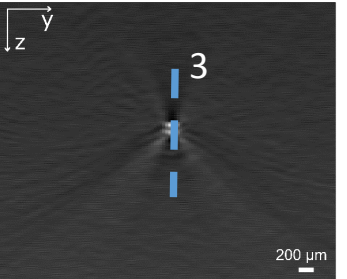

c)

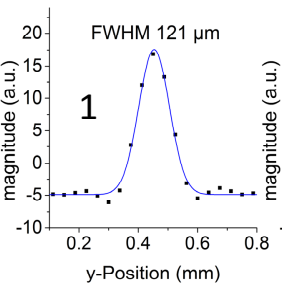

d)

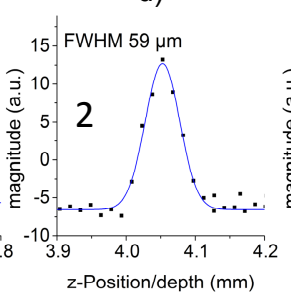

e)

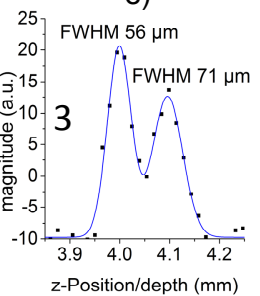

Figure 10. z-y cross section through the reconstructed 3-D image at x-positions where the nylon string pair is separated (a) and in close proximity (b). Panels (c) - (e) show image intensity profiles corresponding to the dashed lines in Figure 10(a) and (b). 


\section{DISCUSSION AND CONCLUSIONS}

In summary, a Fabry-Perot polymer film sensor consisting of hard dielectric mirrors and a Parylene $\mathrm{C}$ spacer deposited onto a polymer substrate was successfully developed, characterized, and applied to PA tomography. The hard dielectric mirror materials resulted in acoustic impedance mismatches that affect the propagation of ultrasound ${ }^{4}$. To describe the transduction mechanisms, a numerical model of the sound propagation through the sensor was developed and used to predict the frequency response. The modelled frequency response exhibited a resonant peak at $25 \mathrm{MHz}$, which was validated experimentally. The ITF fringe width, visibility and sensitivity were comparable to those measured in FP sensors with soft dielectric mirrors ${ }^{4}$. It was shown that the sensor is capable of 3-D tomographic imaging of tissue phantoms with high spatial resolution. In future work, the resolution and directivity of the sensor will be measured and its capability to obtain in vivo PA images will be demonstrated. Due to the inherently robust and non-hygroscopic hard dielectricmaterials and the sensitivity and frequency response of FP interferometer, this sensor has the potential to substitute those currently made using soft dielectric materials.

\section{ACKNOWLEDGEMENTS}

This project was supported by the Deutsche Forschungsgemeinschaft (DFG project grant LA3273/1-1) and the European Research Commission (ERC Starting Grant 281356).

\section{REFERENCES}

[1] Ling, T., Chen, S.-L.., Guo, L. J., "High-sensitivity and wide-directivity ultrasound detection using high Q polymer microring resonators.,” Appl. Phys. Lett. 98(20), 204103 (2011).

[2] Zhang, C., Ling, T., Chen, S.., Guo, L. J., "Ultrabroad Bandwidth and Highly Sensitive Optical Ultrasonic Detector for Photoacoustic Imaging” (2014).

[3] Beard, P. C., Perennes, F.., Mills, T. N., "Transduction mechanisms of the Fabry-Perot polymer film sensing concept for wideband ultrasound detection,” IEEE Trans. Ultrason. Ferroelectr. Freq. Control 46(6), 1575-1582 (1999).

[4] Zhang, E., Laufer, J.., Beard, P., "Backward-mode multiwavelength photoacoustic scanner using a planar FabryPerot polymer film ultrasound sensor for high-resolution three-dimensional imaging of biological tissues.," Appl. Opt. 47(4), 561-577 (2008).

[5] Hajireza, P., Krause, K., Brett, M.., Zemp, R., "Glancing angle deposited nanostructured film Fabry-Perot etalons for optical detection of ultrasound.," Opt. Express 21(5), 6391-6400 (2013).

[6] Treeby, B. E.., Cox, B. T., "k-Wave: MATLAB toolbox for the simulation and reconstruction of photoacoustic wave fields," J. Biomed. Opt. 15(2), 021314 (2010).

[7] Capilla, J., Olivares, J., Clement, M., Sangrador, J., Iborra, E.., Devos, a., "Characterization of amorphous tantalum oxide for insulating acoustic mirrors,” Proc. IEEE Int. Freq. Control Symp. Expo. (2011).

[8] Waks Serra, M., Carbone, N., Di Rocco, H., García, H., Iriarte, D., Pomarico, J.., Ranea-Sandoval, H., "Diffuse light transmission profiles obtained using CW: A comparative analysis with time resolved experiments," Optik (Stuttg). 125(14), 3507-3513, Elsevier GmbH. (2014). 